\title{
The limits of SARS-CoV-2 predictability
}

\author{
The future of SARS-CoV-2, including the possibility of elimination and eradication, remains uncertain, but \\ much hinges on characteristics of SARS-CoV-2 immunity. The next few months to a year is a critical period for \\ understanding these characteristics.
}

\section{Rachel E. Baker, Sang Woo Park, Caroline E. Wagner and C. Jessica E. Metcalf}

A $s$ vaccine rollout has expanded in many countries, so have hopes that the 'end of the pandemic' is near. Yet ongoing devastating outbreaks, fuelled by highly transmissible variants, show that hopes for an imminent pandemic end are short-sighted. Further complicating the efforts to predict dynamics on either a short, medium or long term is considerable uncertainty about human behaviour, government interventions and vaccine roll outs, all of which vary across and within countries. Crucially, the strength, range and duration of natural and vaccinal SARS-CoV-2 immunity remain uncertain and the next few months to a year presents a critical period for building understanding of SARS-CoV-2 immunity. While current vaccines appear to be effective against novel strains ${ }^{1}$, particularly in preventing severe disease, their future efficacy remains unclear. Looking forward, a key question is whether SARS-CoV-2 will become endemic, and how the dynamics of endemicity might unfold. The answer depends on core characteristics of immunity as well as the scope of governmental and international responses. To develop expectations for the longer-term and post-pandemic period for SARS-CoV-2, we identify how these levers might determine epidemic trajectories and whether or not eradication of this pathogen is possible.

\section{The landscape of immunity over the longer term}

To bound expectations for the future, we consider the range of immune consequences of infection (Fig. 1). For some pathogens, natural or vaccinal immunity is 'perfect' or 'sterilizing', that is, prevents future infections entirely (for example, measles). In others, immunity is lost, either as a result of pathogen evolution or because immunity itself wanes (for example, typhoid). In extreme cases (for example, influenza), antigenic evolution (to escape host immunity) can lead to secondary infections that may be as severe and as transmissible as the primary infection. However, primary infections from many pathogens, such as RSV (respiratory syncytial virus) and the endemic coronaviruses, lead to 'partial immunity', which does not necessarily prevent secondary infections but still limits their transmissibility and/or severity. For example, secondary infections of RSV are expected to be less transmissible and less severe than a primary RSV infection ${ }^{2}$.

Evidence from the four endemic coronaviruses provide clues as to the possible future of SARS-CoV-2. The first infection with an endemic coronavirus happens in childhood and appears relatively mild. Partial immunity does not limit reinfection but does limit the severity of secondary infections ${ }^{3}$, meaning that individuals who are re-infected at an older age - typically the high-risk group for SARS-CoV-2 - tend to have milder outcomes. If SARS-CoV-2 follows a similar pathway, vaccination at a younger age could replace the primary infection. Depending on the duration of vaccinal immunity, SARS-CoV-2 could then circulate seasonally as a mild, so-called 'common-cold' type outbreak. Within the coming year, it should be increasingly clear from ongoing sampling of vaccinated and previously infected populations, the extent to which SARS-CoV-2 will follow the trajectory of the endemic coronaviruses. Tracking both the individual-level immune responses in focal cohorts (including previously infected and vaccinated individuals), alongside surveillance of case numbers in populations with tracked vaccination and infection history as novel variants emerge, will provide crucial clues to what the future holds. This, in turn, is likely to shape the degree to which elimination or eradication are realistic policy goals.

\section{Is eradication possible?}

The dynamics of immunity will dictate patterns of endemicity in the absence of intervention, but also the pathway to local elimination and global eradication. Different immunity scenarios will require different degrees of global coordination and effort to achieve eradication, illustrated in Fig. 2.

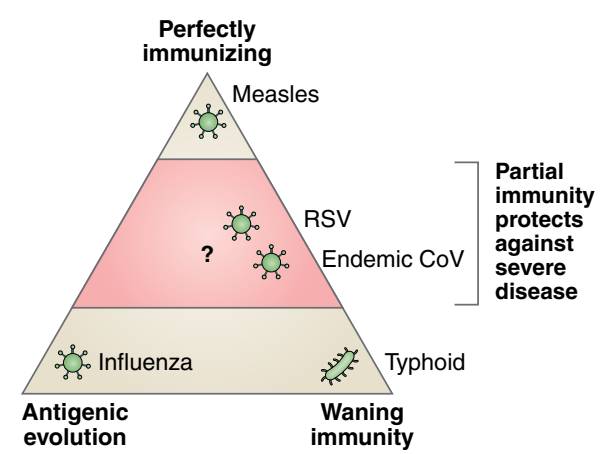

Fig. 1 | Possible immune scenarios for pathogens including SARS-CoV-2. The three corners

represent extremes but many pathogens occupy the middle ground.

For pathogens that evolve rapidly to evade host immunity (for example, influenza), local elimination and global eradication is distinctly difficult. Advances in developing universal vaccines, for example, that work against all influenza strains, provide some hope for eradication of these diseases ${ }^{4}$. If SARS-CoV-2 evolution exhibits similar characteristics, then seasonally updated SARS-CoV-2 vaccines may be required to minimize mortality and morbidity, and a universal vaccine may be necessary for eradication. However, the fact that current SARS-CoV-2 vaccines appear effective across strains, particularly in terms of preventing severe disease, is a source of cautious optimism.

Infections that provide partial or waning immunity (rather than displaying evolution of immune escape) may be easier to control. If immunity wanes, booster vaccines at scheduled intervals could help minimize the risk of a severe outbreak. If immunity is partial, vaccinations or exposure during childhood could minimize the risk of severe cases. However, relying on more frequent vaccination schedules presents further problems. New infrastructure may be required to ensure vaccines consistently reach vulnerable populations across the globe, at regular intervals. Issues of vaccine equity, already apparent, will continue unless 


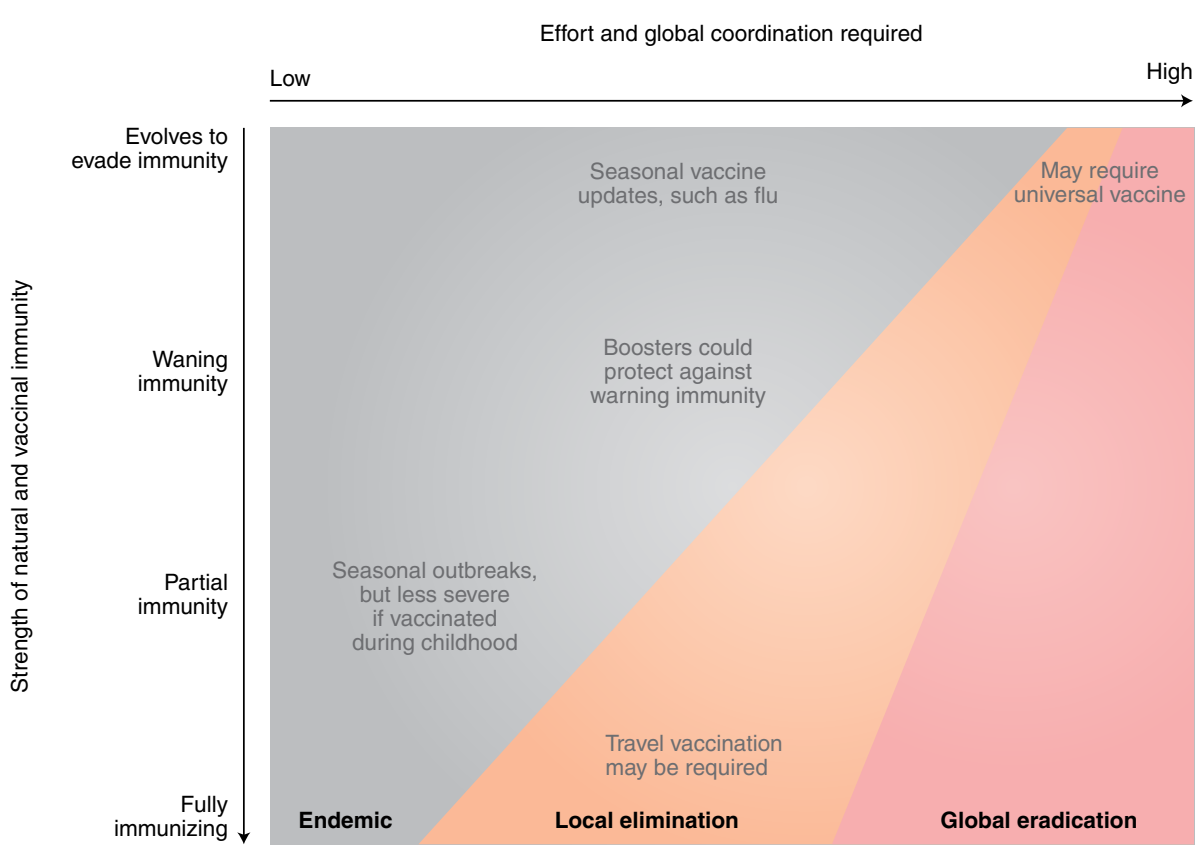

Fig. 2 | Immunity and eradication. Schematic showing the hypothesized effort required to eliminate (orange) and eradicate (red) SARS-CoV-2 under different immunity scenarios.

a global, inclusive approach to vaccine distribution is taken ${ }^{5}$.

If vaccines and natural infections prove to be fully immunizing, then there is a greater hope for local elimination and eventually, global eradication. In this scenario, sufficient numbers of individuals will need to be vaccinated to limit local transmission ${ }^{6}$. Vaccinations for travel into regions with lower coverage will also help limit spread across borders. Despite this promise, the pathway to global eradication is likely to be long. Lessons from ongoing efforts to eradicate polio, or the frequent resurgence of measles after local elimination, speak to the difficulty in achieving these goals. The exact outcome will further depend on other factors, such as vaccine hesitancy, heterogeneity in population structure and intrinsic infectiousness of the pathogen. As such, even in the longer term, a period of SARS-CoV-2 endemicity seems likely.

\section{Drivers of endemic SARS-CoV-2 dynamics}

If an endpoint of continued circulation (endemicity rather than eradication) seems likely, this still leaves us with questions about the range of outbreak sizes, their intensity and seasonality. Surprisingly, some basic epidemiological parameters for predicting these dynamical features are still uncertain. For example, $R_{0}$, the reproductive number, which captures the infectiousness of the pathogen, is typically measured from the growth of the epidemic and is harder to estimate once non-pharmaceutical interventions (NPIs) are in place. Similarly, changes to $R_{0}$ for evolved SARS- CoV-2 variants are difficult to ascertain given simultaneous changes to behaviour and interventions. It is not yet clear whether there is an evolutionary limit to strain infectiousness. To date, structural changes to the SARS-CoV-2 spike furin cleavage site $^{7}$ as well as enhanced binding of the receptor binding domain to the human ACE2 receptor ${ }^{8}$ have been associated with enhanced transmissibility in variant strains, but in the longer term, transmission increases may saturate and viral evolution may modulate other aspects of disease transmission including host susceptibility. Nevertheless, any present or future changes to $R_{0}$ will affect long-term epidemic dynamics, including the intensity of outbreaks and the age-structure of infections.

The transmission of many respiratory pathogens varies seasonally, driven either by climatic factors or seasonal changes in behaviour such as schooling. The role of climate in driving transmission of SARS-CoV-2 is currently unclear: high susceptibility during the early pandemic likely limited any climate effect $^{9}$, and statistical analyses of the climate-SARS-CoV-2 link have been confounded by trends in the data and regional differences in reporting and control measures. This has not been helped by the relatively short case time series (that is, just over a year's worth of data) compared to typical climate-disease studies that look for climate links over many seasons. An alternative line of evidence comes from the four endemic coronaviruses, which exhibit seasonal wintertime outbreaks. It is possible that SARS-CoV-2 will follow suit. Disentangling the climate drivers of SARS-CoV-2 will become easier over time as both longer time series are available, and susceptibility declines 9 .

A further question is the extent to which SARS-CoV-2 endemic dynamics will be affected by interactions with other circulating pathogens, including the endemic coronaviruses. Both modelling and laboratory work implies a degree of cross-immunity between coronaviruses ${ }^{10-12}$. The NPIs put in place to limit the spread of SARS-CoV-2 have also limited the circulation of many other pathogens, such that infection interactions have not been observed in current case trajectories ${ }^{13}$. However, as NPIs are relaxed, signatures of cross-species interactions will likely become increasingly visible.

Beyond cross-immunity with other pathogens, the longitudinal trajectory of immunity, as depicted in Fig. 1, will play a crucial role in determining SARS-CoV-2 endemic dynamics ${ }^{14}$. For immunizing infections, susceptibility is driven by birth rates, and infections may be concentrated in younger age groups. For infections with waning immunity or antigenic evolution, susceptibility is driven by the rate at which immunity wanes or the rate the pathogen evolves as well as characteristics of secondary infections. The disease dynamics of pathogens with high rates of antigenic evolution are particularly hard to predict: evolved strains may have variable transmission rates and manifest variable immune responses. An analogy can be made with influenza, where the size and intensity of the seasonal influenza peak is typically very difficult to forecast ${ }^{15}$.

The future course of SARS-CoV-2 remains uncertain. The next few months to a year represents a critical time where we will begin to develop an understanding of key parameters, such as the strength and duration of vaccinal and natural immunity, the seasonality of transmission and the possible interaction of SARS-CoV-2 with other circulating pathogens. In combination, these parameters will allow improved prediction of both long-term SARS-CoV-2 epidemic dynamics, as well as the likelihood of elimination and eradication. An area of particular focus will be the rate of antigenic evolution and the extent to which vaccines 
remain protective against evolved strains. In all scenarios, rapid and equitable distribution of vaccines presents the greatest hope for minimizing future severe outbreaks.

Rachel E. Baker D1,2凶, Sang Woo Park (D), Caroline E. Wagner ${ }^{3}$ and

\section{Jessica E. Metcalf (iD) 1,4}

${ }^{1}$ Department of Ecology and Evolutionary Biology, Princeton University, Princeton, NJ, USA. ${ }^{2}$ Princeton High Meadows Environmental Institute, Princeton University, Princeton, NJ, USA. ${ }^{3}$ Department of Bioengineering, McGill University, Montreal, Quebec, Canada. ${ }^{4}$ Princeton School of Public and International Affairs, Princeton University, Princeton, NJ, USA.

$凶_{e-m a i l: \text { racheleb@princeton.edu }}$
Published online: 12 July 2021

https://doi.org/10.1038/s41559-021-01514-Z

$\square \quad$ References

1. Liu, Y. et al. N. Engl. J. Med. 384, 1466-1468 (2021).

2. Pitzer, V. E. et al. PLoS Pathog. 11, el004591 (2015).

3. Lavine, J. S., Bjornstad, O. N. \& Antia, R. Science 371, 741-745 (2021).

4. Nachbagauer, R. et al. Nat. Med. 27, 106-114 (2021)

5. Katz, I. T., Weintraub, R., Bekker, L.-G. \& Brandt, A. M. N. Engl. J. Med. 384, 1281-1283 (2021).

6. Metcalf, C. J. E., Ferrari, M., Graham, A. L. \& Grenfell, B. T. Trends Immunol. 36, 753-755 (2015).

7. Rambaut, A. et al. Preliminary Genomic Characterisation of an Emergent SARS-CoV-2 Lineage in the UK Defined by a Novel Set of Spike Mutations (2020); https://go.nature.com/3yl6kMh

8. Zahradník, J. et al. Preprint at bioRxiv https://doi. org/10.1101/2021.01.06.425392 (2021).

9. Baker, R. E., Yang, W., Vecchi, G. A., Metcalf, C. J. E. \& Grenfell, B. T. Nat. Commun. 12, 846 (2021).
10. Kissler, S. M., Tedijanto, C., Goldstein, E., Grad, Y. H. \& Lipsitch, M. Science 368, 860-868 (2020).

11. Ng, K. W. et al. Science 370, 1339-1343 (2020).

12. Braun, J. et al. Nature 587, 270-274 (2020).

13. Baker, R. E. et al. Proc. Natl Acad. Sci. USA 117, 30547-30553 (2020).

14. Saad-Roy, C. M. et al. Science 370, 811-818 (2020).

15. Biggerstaff, M. et al. Epidemics 24, 26-33 (2018).

Acknowledgements

R.E.B. is supported by the Cooperative Institute for

Modeling the Earth System. C.J.E.M. is supported by the

Centre for Health and Wellbeing at Princeton University.

Author contributions

R.E.B., S.W.P., C.E.W. and C.J.E.M. contributed to the writing and conceptualization of this manuscript.

Competing interests

The authors declare no competing interests. 